International Journal of Case Reports
(ISSN:2572-8776)

\title{
Acute soft head syndrome and a mini review of bone and neurologic complications in patients with sickle cell disease
}

\author{
Ehab Hanafy ${ }^{1}$, Saad Al Amri ${ }^{2}$, AbdulKareem Alenazi ${ }^{2}$, Amal Al Balawi ${ }^{2}$, Nadia Abdullah ${ }^{3}$, \\ Nazim Faisal ${ }^{1}$, Gihan Mahmoud ${ }^{1}$ \\ ${ }^{1}$ Prince Sultan Oncology Center, King Salman Armed Forces Hospital, Tabuk, Kingdom of Saudi \\ Arabia. \\ ${ }^{2}$ Pediatric Department, King Salman Armed Forces Hospital, Tabuk, Kingdom of Saudi Arabia. \\ ${ }^{3}$ Faculty of Medicine, Tabuk University, Tabuk, Kingdom of Saudi Arabia.
}

\section{ABSTRACT}

Introduction: Acute soft head syndrome is a rare complication in patients with sickle cell disease (SCD) that is characterized by Ehab Hanafy

progressive skull pain and swelling, it is usually related to skull Prince Sultan Oncology Center, bone infarction and full recovery is achieved with conservative King Salman Armed Forces Hospimanagement. This case represents a very rare association of tal, Tabuk 100, Kingdom of Saudi sickle cell disease and acute soft head syndrome that necessi- Arabia.

tates to be differentiated from other bone and neurologic presentations occurring in this entity of patients.

How to cite this article:

Case: We report a rare case of an 11 years old Saudi male paEhab Hanafy, Saad Al Amri, Abtient with sickle cell disease who developed acute soft head dulKareem Alenazi, Amal Al Balasyndrome that presented with severe headache. An underlying osteomyelitis of skull and lower limb bones couldn't be excluded wi, Nadia Abdullah, Nazim Faisal, Gihan Mahmoud. Acute soft head and the patient had full recovery after conservative treatment. syndrome and a mini review of Conclusion: Acute soft bone syndrome seldomly occurs in SCD, bone and neurologic complications however, its presentations mimics those of bone and neurologic complications so differentiation is essential in order to set up a clear plan of management. in patients with sickle cell disease. International Journal of Case Reports, $20194: 80$

Keywords: Sickle cell disease, acute soft head syndrome, stroke, headache, osteomyelitis

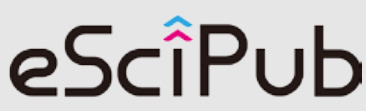

eSciPub LLC, Houston, TX USA.

Website: https://escipub.com/ 


\section{Background}

Sickle cell disease (SCD) is a common recessively inherited hemoglobinopathy that is characterized by the production of abnormal HB $S$ which is readily polymerized leading to erythrocyte stiffness and vaso-occlusion. These changes will ultimately cause microcirculation obstruction and tissue ischemia (1). Acute soft bone syndrome is a rare complication in SCD with a poorly understood pathogenesis, and its presentation often mimics those of neurologic and bone complications. It manifests with progressive skull swelling, pain and tenderness. Imaging is the mainstay of diagnosis and management is mostly conservative with full recovery (2). Bone and neurologic involvement are among the most common complication in SCD and are responsible for the majority of hospital admission. They present with a wide spectrum of manifestations with different grades of severity. For instance, bone involvement in SCD is the most common complication and occurs as early as first few months of life in the form of dactylitis. Skeletal complications can be either acute as in vaso-occlusive crises, osteomyelitis, and septic arthritis or occurs in a more chronic form that leads to variable disabilities as in avascular necrosis of femoral head and osteopenia (3). Contrarily, neurologic complications are serious enough to cause a wide range of morbidities in SCD. These can present with simple headache or with more severe manifestations like increased intracranial tension, hemiparesis, dysarthria and blindness. There is a range of recognized neurologic complications including ischemic, hemorrhagic and silent stroke. Other rare problems such as neuropathic pain, posterior reversible encephalopathy syndrome, and brain atrophy can occur in patients with SCD (4). Special considerations are given to those patients who are vulnerable to develop psychosis, delirium, or neurocognitive disorders (5).

\section{Case presentation}

This is an 11-year old Saudi male patient presented with severe headache mainly in the temporal areas of both sides associated with jaw and back pain. Temperature was $37.8^{\circ} \mathrm{C}$ at presentation, oxygen saturation was $97 \%$ room air, blood pressure was normal for age $(110 / 70)$ and there was no tachycardia ( 75 beats $/ \mathrm{min}$ ). The headache was severe, continuous and not relieved by simple analgesia.

He was diagnosed as SCD at the age of 2 years. Both parents are consanguineous, the father has sickle cell trait whereas the mother has sickle cell disease, 3 of his siblings were diagnosed with sickle cell disease while one sibling was diagnosed with sickle cell trait. There was no history of hypertension, diabetes or other conditions of medical concerns, he has history of splenectomy at age of 4 years because of a major attack of splenic sequestration. There was no history of drug allergies. Developmental history is compatible with his age and vaccination is up to date. He was on regular follow up at our Pediatric hematology clinic and on regular hydroxyurea, folic acid supplements and oral penicillin.

On examination, the head was mildly swollen but severely tender bilaterally, there was mild swelling at his lower back and left lower limb associated with severe tenderness and hotness, there was no hepatomegaly, ear/nose/throat (ENT) examination was unremarkable, initial neurologic examination was unremarkable with normal motor power, sensations and reflexes. Subsequently, he was admitted at the hospital, he started on full supportive care including double maintenance intravenous fluids, morphine infusion and broad-spectrum antibiotics. Routine labs and an urgent hemoglobin electrophoresis were done and showed HBS of $88 \%$ (table 1).

During his first day at the hospital, computed tomography (CT) scan of the brain showed patchy sclerotic area of the left high parietal bone with associated small overlying scalp soft tissue sheet and a possibility of acute soft head syndrome. We advised against performing exchange transfusion at that time as no clinical and imaging findings suggestive of stroke. Trans 
cranial doppler (TCD) was also done and reported as normal.

The child showed remarkable improvement of headache in the next few days of admission but was still having moderate leg pain. Magnetic resonance imaging (MRI) of the brain was done and showed an abnormal signal in the intramedullary areas of the posterior aspect of both parietal bones eliciting low/intermediate signal on T1 and high signal on T2 with no appreciable enhancement in the post-contrast series. On the right side, small areas of cortical rarefaction were noted with associated enhancing extra-osseous, extra-cranial scalp (about $5 \mathrm{~mm}$ along maximum thickness) and intra-cranial, extra-dural (about $8 \mathrm{~mm}$ along its maximum thickness) soft tissue sheets, the latter was seen indenting the underlying brain parenchyma. On the left side, associated extracranial scalp enhancing soft tissue sheet was seen measuring about $5 \mathrm{~mm}$ along its maximum thickness. Another midline parietal extracranial scalp soft tissue sheet is seen measuring about $8 \mathrm{~mm}$ along its maximum thickness. This picture was suggestive of acute soft head syndrome (Calvarial bone infarction with extravasated hemopoietic tissue and blood in the subgaleal and epidural spaces however, the possibility of osteomyelitis could not be ruled out (figure 1).

MRI lower limbs showed minimal periosteal reaction that was seen at the left antero-medial aspect of middle tibia (about $5 \times 37 \mathrm{~mm}$ ) with intact underlying cortex, it displayed low signal at T1, intermediate at T2 and high at STIR and exhibited faint marginal enhancement. The MRI concluded the possibility of osteomyelitis due to the presence of periosteal reaction but couldn't rule out bone infarction as well (figure 2).

Orthopedic and infectious disease consultations were done and agreed upon starting the patient on full regimen of broad-spectrum antibiotics towards osteomyelitis despite the wellappreciated clinical improvement of the patient.

\begin{tabular}{|l|l|l|}
\hline & Value & Normal value \\
\hline SODIUM & 142 & $132-146 \mathrm{mmol} / \mathrm{L}$ \\
\hline POTASSIUM & 4.5 & $3.6-5.0 \mathrm{mmol} / \mathrm{L}$ \\
\hline CHLORIDE & 101 & $98-107 \mathrm{mmol} / \mathrm{L}$ \\
\hline ENZYMATIC BIOCARBONATE & 20 & $22-29 \mathrm{mmol} / \mathrm{L}$ \\
\hline UREA NITROGEN & 1.3 & $1.6-4.6 \mathrm{mmol} / \mathrm{L}$ \\
\hline CREATININE & 44 & $20-70 \mu \mathrm{mol} / \mathrm{L}$ \\
\hline TOTAL PROTEIN & 82 & $64-86 \mathrm{~g} / \mathrm{L}$ \\
\hline ALBUMIN & 42 & $38-56 \mathrm{~g} / \mathrm{L}$ \\
\hline ALP & 176 & $218-499 \mathrm{U} / \mathrm{L}$ \\
\hline AST (SGOT) & 62 & $15-37 \mathrm{U} / \mathrm{L}$ \\
\hline ALT (SGPT) & 36 & $24-49 \mathrm{U} / \mathrm{L}$ \\
\hline TOTAL BILIRUBIN & 66 & $0-14 \mu \mathrm{mol} / \mathrm{L}$ \\
\hline WBC & 18 & $5.0-13.010^{\wedge} 3 / \mu \mathrm{l}$ \\
\hline HEMOGLOBIN & 9.3 & $11.5-14 \mathrm{~g} / \mathrm{dl}$ \\
\hline PLT & 323 & $180-40010^{\wedge} 3 / \mathrm{\mu l}$ \\
\hline RETICS & 13 & $0.5-2.5$ \\
\hline C-REACTIVE PROTIEN & 16.4 & $0.06-0.79 \mathrm{mg} / \mathrm{dl}$ \\
\hline ESR & 50 & $1-20$ \\
\hline & HBA: 0 & $\mathrm{HBA}: 95-98 \%$ \\
\hline HB ELECTROPHOREIS & HBA2:3.3 & HBA2:1.5-3.5\% \\
& HBS:88.8 & HBS:0 \\
\hline
\end{tabular}

Table 1 showing blood work at the time of presentation 


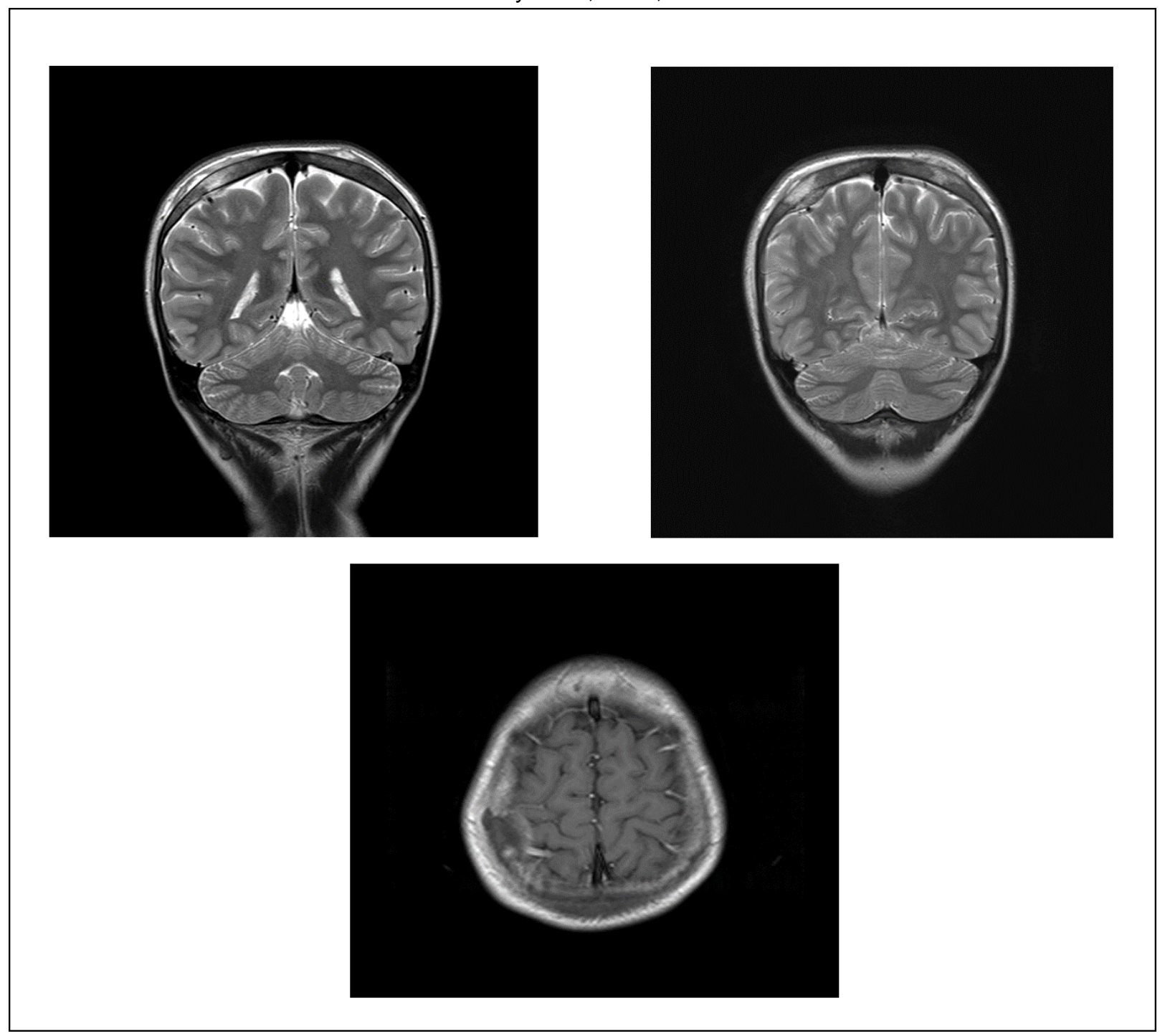

Figure 1 MRI Brain

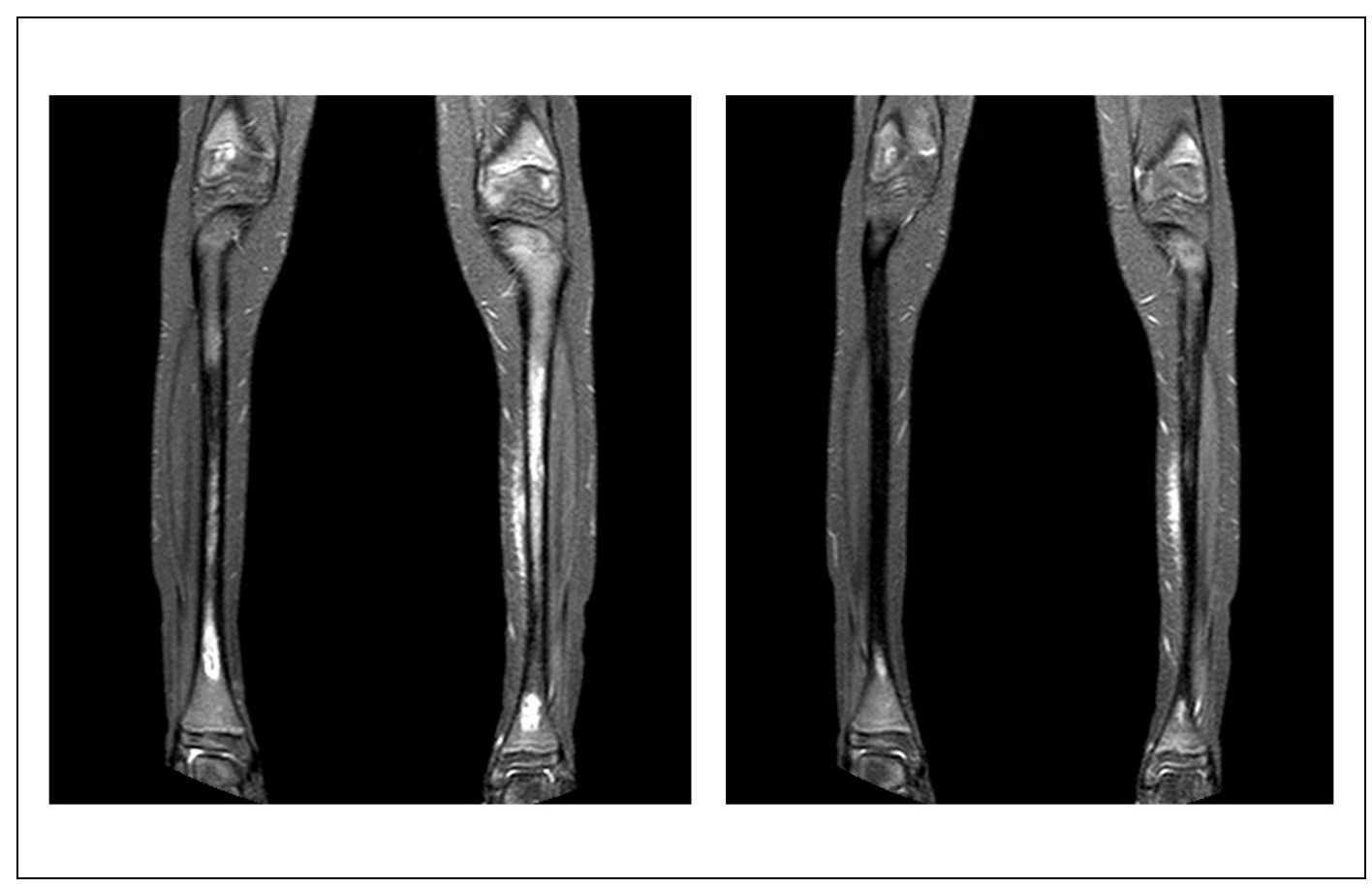

Figure 2 MRI lower limbs

IJCR: https://escipub.com/international-journal-of-case-reports/ 


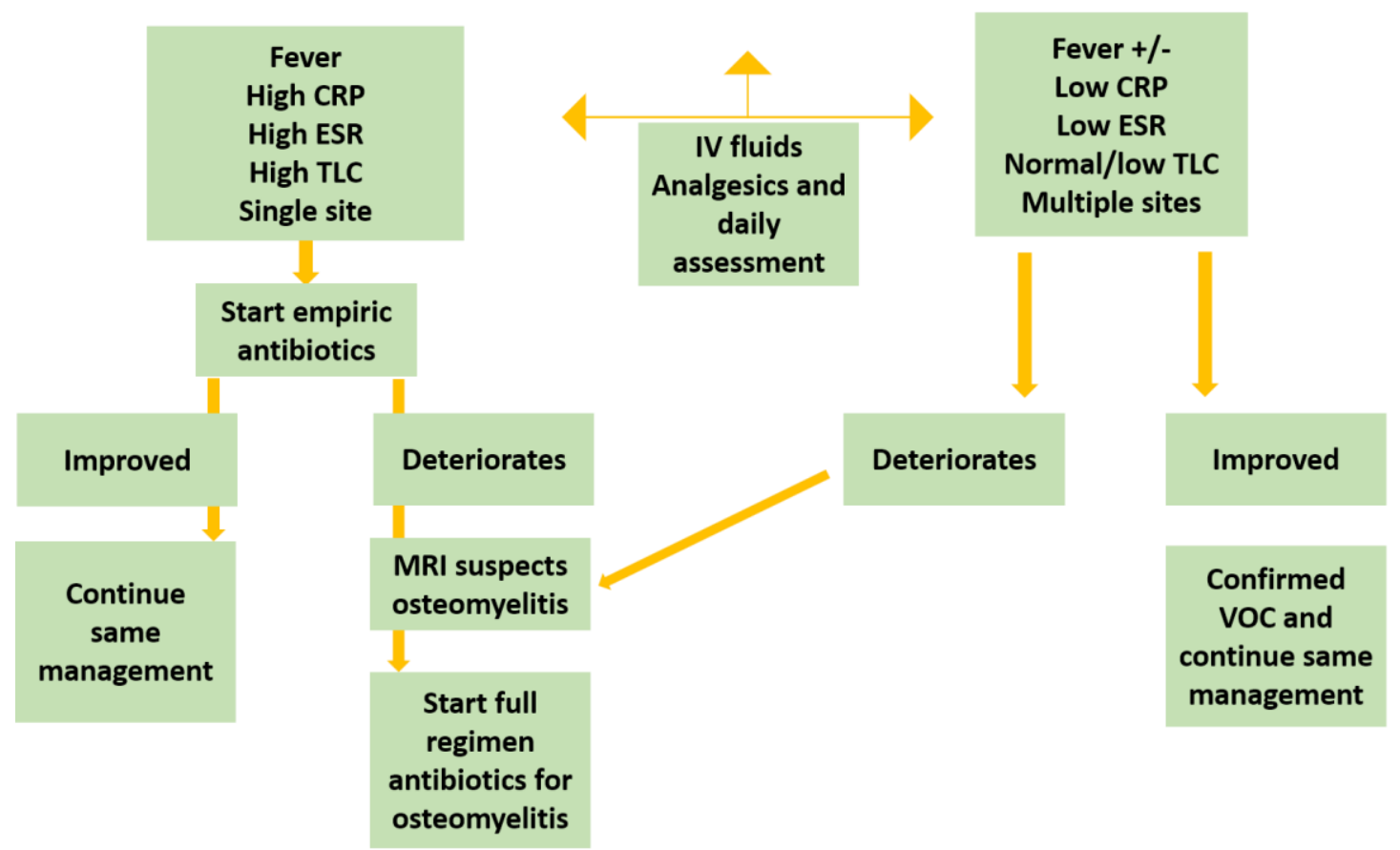

Figure 3 management of SCD patients presenting with painful episode - local hospital guideline

\section{Discussion}

We present a rare case of acute soft head syndrome in a child with sickle cell disease, the child presented with severe headache and skull swelling. Having known that both symptoms are encountered frequently in patients with sickle cell disease due neurologic and bone complications, our report is based on reviewing these complications and the need to differentiate them from this rare presentation.

Sickle cell disease is a common hereditary autosomal recessive disorder in the Kingdom of Saudi Arabia. The reported prevalence for sickle cell trait ranges from $2 \%$ to $27 \%$, and up to $2.6 \%$ will have SCD in some areas. It is characterized by the production of abnormal hemoglobin $S$ which is responsible for a variety of complications that ranges in severity from mild to life threatening events (6).

Acute soft head syndrome is characterized by progressive skull pain and swelling that occurs spontaneously without a recognized history of trauma. It is a rare complication in patients with
SCD and few cases only have been reported in the literature $(7,8,9)$.

The pathophysiology of acute soft head syndrome is not fully understood yet. Nevertheless, few theories have been proposed including a vaso-occlusive crisis leading to bone infarction that will ultimately disrupts the cortical bone and causes periosteal elevation and subsequent bleeding in the subgaleal and epidural spaces (2). Other theory indicates a poor venous drainage that will lead to edema and hemorrhage (10). And third theory which proposes extravasation of blood and marrow in the subgaleal and epidural spaces due to disruptions in the skull cortex caused by bone marrow hyperproliferation (11).

Imaging with CT and MRI is the cornerstone for diagnosis of this condition beside other causes of skull pain and swelling. However, MRI is more sensitive in detecting bone infarctions (12). Both imaging has been done for our reported case and clearly demonstrated the high possibility of acute soft bone syndrome due to the presence 
of calvarial bone infarction with extravasated hemopoietic tissue and blood in the subgaleal and epidural spaces. The management is usually conservative including intravenous fluids and analgesics, with most of the patients achieving full recovery.

Bone involvement is the commonest clinical presentation in sickle cell disease and can occur both in acute setting and as a chronic disability. It is characterized by osteonecrosis, osteomyelitis and arthritis. The bone microcirculation is a common site for erythrocyte sickling leading to infarcts, thrombosis and ischemic necrosis. Vaso-occlusive crises and osteomyelitis are the most common causes of admission in these patients (13). Bone involvement usually presents with pain that can be precipitated by infection, change in climate or psychological stress which can be accompanied sometimes by local tenderness, erythema, warmth, and swelling (14).

The most common affected areas are the lumbosacral spine, knee, shoulder, elbow, and femur. Joint effusions during acute stages are particularly common in the knees and elbows. These findings are very similar to those of osteomyelitis or septic arthritis and it is considered one of the most common diagnostic challenges that faces the practitioners when faced with patients having painful episodes. Saito et al. showed that vaso-occlusive episodes are 50 times more frequent than osteomyelitis in children with sickle cell disease (12). However, in children with SCD presenting with persistent bone pain, fever, elevated CRP and white cell count, osteomyelitis should be suspected and immediate antibiotic treatment should start (15). Furthermore, a study by Berger et al. concluded that swelling in one site associated with fever and pain increased the probability of osteomyelitis in children with sickle cell disease (16). The strategy of starting antibiotic regimens for these patients has reduced the occurrence of osteomyelitis in $98.6 \%$ and septic arthritis in $100 \%$ in one study (15).
Therefore, differentiating painful crisis in SCD from osteomyelitis is a diagnostic dilemma and despite the progress in the development of imaging techniques, a definitive diagnosis of osteomyelitis in SCD depends more on clinical assessment together with some laboratory markers and positive cultures from blood or bone obtained by aspiration or biopsy.

Besides headache as a presenting symptom, our reported case had left leg pain and tenderness as well, his lab works showed a high level of CRP, ESR and high total leukocytic count. This raised suspicious towards osteomyelitis particularly when it couldn't be ruled out with both MRI head and lower limbs. We started him on the full regimen antibiotic therapy for osteomyelitis as per our local guideline (figure 3) despite showing some improvement on initial conservative management including fluids and analgesics.

Dactylitis (hand-foot syndrome) is another form of bone involvement in SCD and is typically the first vaso-occlusive complication to occur. It manifests in children less than 5 years where the small bones of hands and feet are usually affected and can be associated with edema and erythema. Its occurrence before the age of 1 year is predictive of disease severity by the age of 10 years (17)

Some other chronic skeletal changes in SCD include overgrowth of the anterior maxilla that may lead to severe orthodontic and cosmetic problems, flattening of the vertebrae with a characteristic biconcave deformity called codfish vertebrae that may cause chronic back pain in older patients (18). The major chronic bone complication is avascular necrosis of the head of femur that occurs more frequently in older patients. Its pathophysiology is poorly understood but can be attributed to limited blood supply in the femoral head that promotes sludging in marrow sinusoids, marrow necrosis, healing with increased intramedullary pressure, articular surface necrosis, bone resorption, and eventually epiphyseal collapse (19). 
Overall, the treatment of bone involvement in SCD is mostly symptomatic unless an infectious source is proven. Further studies are required to understand the pathophysiological mechanisms involved in these complications and propose more tailored and specific therapies.

On the other hand, neurologic complications are an important cause of morbidity in SCD. These patients usually manifest with strokes that can simply present with headache. Thus, thorough investigations should be carried out in order not to miss other contributing causes of headache which will not urge invasive interventions as in stroke.

Stroke is defined as acute neurologic insult caused by vascular occlusion or hemorrhages that result in ischemia and focal neurologic symptoms or signs lasting more than 24 hours, whereas silent stroke is an MRI evidence of cerebral ischemia in the absence of focal neurologic deficits. It is estimated that $11 \%$ of patients with SCD would develop stroke by age of 20 years and $24 \%$ by 45 years. Moreover, recurrent stroke occurs in two third of SCD within 2-3 years (20). Ischemic strokes are secondary to vasculopathy and arterial stenosis and are predisposed by transient ischemic attacks, hypertension and nocturnal hypoxemia (21), while hemorrhagic strokes results from subarachnoid hemorrhage, intraventricular hemorrhage or as combination and are associated with older ages, low hemoglobin and prior blood transfusion within 2 weeks (22). Other than headache, patients with stroke can present with motor disabilities (hemiparesis, abnormal gait), focal seizures, speech defect or signs of increased intracranial tension.

Patients with neurological symptoms should be evaluated by CT and MRI to distinguish thrombosis from hemorrhage. Immediate exchange transfusion to lower the $\mathrm{HbS}$ level to less than $30 \%$ is required. However, Patients with hemorrhage may require different surgical interventions.

Our patient's main symptom was headache, even before the skull swelling manifested. This symptom shouldn't be taken lightly in patients with SCD; therefore, an urgent CT scan of the brain was requested as the headache was severe and progressive. According to our protocol, if the CT scan of the brain is normal and the patient is still having progressive headache, we will either proceed with an MRI brain and MR angiography - if feasible or we will carry out immediate exchange transfusion in order to prevent any impending stroke. Fortunately, the CT brain ruled out any hemorrhage but suggested the diagnosis of acute soft head syndrome. Thus, exchange transfusion was avoided and MRI brain was done later and confirmed the presence of subgaleal and epidural collections suggesting acute soft head syndrome.

Finally, prevention, early detection and treatment of neurologic complications is important. Transcranial Doppler (TCD) ultrasonography has been shown to be an effective screening tool for identification of stroke risk. TCD screening should begin at 2 years of age and continue annually into the late teenage years. Increased blood flow velocity due to stenosis can be detected by TCD ultrasonography in asymptomatic patients, and flow rates in excess of $200 \mathrm{~cm} / \mathrm{s}$ correlate with a high risk of stroke (3).

\section{Conclusion}

Bone and neurologic manifestations are common in patients with SCD. However, these manifestations can overlap with other rare presentations and every effort should be done to differentiate them in order to have a clear management plan without the need of unnecessary interventions. Acute soft head syndrome should be ruled out in patients with SCD who present with headache, skull tenderness or swelling, its course is benign and can be managed successfully using conservative measures.

\section{Declarations}

\section{Ethics approval and consent to participate:} not applicable 
Consent for publication: Written informed consent was obtained from the patient's legal guardian for publication of this case report and any accompanying images. A copy of the written consent is available for review by the Editor-inChief of this journal.

\section{Availability of data and supporting materials: not applicable}

Competing interests: the author(s) declared no potential conflicts of interest with respect to the research, authorship, and/or publication of this article.

Funding: the author(s) received no financial support for the research, authorship, and/or publication of this article.

Authors' contribution: $\mathrm{EH}$ - Substantial contributions to conception and design, acquisition of the data, drafting the article, revising it critically for important intellectual content. SA - contributed through acquisition of the data, drafting the article. AE - contributed through acquisition of the data, revising the article. $A B$ - revised it critically for important intellectual content. NA - contributed through acquisition of the data, revising the article. GM revised it critically for important intellectual content.

\section{Acknowledgements: not applicable}

\section{References}

1. Hassan A. Al-Jafar, Raed Alroughani, Thamer A.Abdullah, and Fatma Al-Qallaf. Neurological Complications in Sickle Cell Disease. International Journal of Clinical and Experimental Neurology, vol. 4, no. 1 (2016): 918. doi: 10.12691/ijcen-4-1-2.

2. Arends S, Coebergh JA, Kerkhoffs JL, et al (2011) Severe unilateral headache caused by skull bone infarction with epidural haematoma in a patient with sickle cell disease. Cephalalgia31:1325-28.

3. Hoffbrand, A. V, Higgs, Douglas R, Keeling, David, Mehta, Atul B. (2016) Postgraduate Haematology. 7th edition. Blackwell Publishing Ltd., Oxford, UK.

4. Ekaterini Solomou, Pantelis Kraniotis, Alexandra Kourakli, and Theodore Petsas. Extent of Silent Cerebral Infarcts in Adult SickleCell Disease Patients on Magnetic Resonance Imaging: Is There a Correlation with the Clinical
Severity of Disease? Hematol Rep. 2013 Jan 25; 5(1): 8-12.

5. Berkelhammer LD, Williamson AL, Sanford SD, Dirksen CL, Sharp WG, Margulies AS, et al. Neurocognitive sequelae of pediatric sickle cell disease: a review of the literature. Child Neuropsychol 2007;13:120-131.

6. Jastaniah W. Epidemiology of sickle cell disease in Saudi Arabia. Ann Saudi Med. 2011;31(3):289293. doi:10.4103/0256-4947.81540.

7. Akodu SO, Njokanma OF, Diaku-Akinwumi IN, Ubuane PO, Adediji UO. Acute soft head syndrome in children with sickle cell anaemia in lagos, Nigeria. Indian J Hematol Blood Transfus. 2014;30(Suppl 1):67-69. doi:10.1007/s12288013-0251-6.

8. Al-Dabbous IA, Al-Jama AH (1996) Acute soft head syndrome in sickle cell disease (A case report). Saudi Med J17:804-07.

9. Fortin-Boudreault $R$, Story $E$, Simpson E, Johnston D, Chretien C (2016) Spontaneous Subgaleal Hematoma: An Unusual Complication of Sickle Cell Disease. J Clin Case Rep 6: 681. doi:10.4172/2165-7920.1000681.

10. Kalala Okito JP, Van Damme O, Calliauw L. (2004) Are spontaneous epidural haematoma in sickle cell disease a rare complication? A report of two new cases. Acta Neurochir (Wien)146:407-10.

11. Dahdaleh NS, Lindley TE, Kirby PA, et al. (2009) A. J Neurosurg Pediatr4:532-35

12. Saito N, Nadgir RN, Flower EN, Sakai O (2010) Clinical and radiologic manifestations of sickle cell disease in the head and neck. Radiographics 30: 1021-1034.

13. da Silva Junior GB, Daher Ede F, da Rocha FA. Osteoarticular involvement in sickle cell disease. Rev Bras Hematol Hemoter. 2012;34(2):156-164. doi:10.5581/15168484.20120036

14. Redwood AM, Williams EM, Desal $P$, et al: Climate and painful crisis of sickle-cell disease in Jamaica. Br Med J 1:66, 1976.

15. Fontalis A, Hughes $K$, Nguyen MP, et al. The challenge of differentiating vaso-occlusive crises from osteomyelitis in children with sickle cell disease and bone pain: A 15-year retrospective review. J Child Orthop. 2019;13(1):33-39. doi:10.1302/1863-2548.12.180094

16. Berger E, Saunders N, Wang L, Friedman JN (2009) Sickle cell disease in children: differentiating osteomyelitis from vaso-occlusive crisis.Arch Pediatr Adolesc Med 163: 251-255. 
17. Miller ST, Sleeper LA, Pegelow $\mathrm{CH}$, et al: Prediction of adverse outcomes in children with sickle cell disease. N Engl J Med 342:83, 2000.

18. Diggs LW: Bone and joint lesions in sickle-cell disease. Clin Orthop Relat Res 52:119, 1967.

19. Milner PF, Kraus AP, Sebes JI, et al: Sickle cell disease as a cause of osteonecrosis of the femoral head. N Engl J Med 325:1476, 1991.

20. Strouse, John J., Sophie Lanzkron, and Victor Urrutia. The epidemiology, evaluation and treatment of stroke in adults with sickle cell disease. Expert review of hematology 4.6 (2011): 597-606.

21. Webb, Jennifer, and Janet L. Kwiatkowski. Stroke in patients with sickle cell disease. Expert review of hematology (2013).

22. Switzer, Jeffrey A., et al. Pathophysiology and treatment of stroke in sickle-cell disease: present and future. The Lancet Neurology 5.6 (2006): 501-512. 